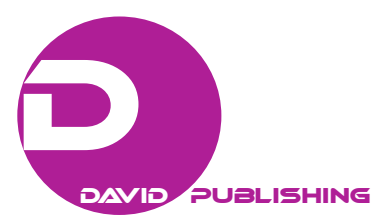

\title{
GPS Signal Correction to Improve Vehicle Location and Related Emission Evaluation
}

\author{
L. Della Ragione \\ Istituto Motori CNR, via Marconi 8 - 80125 Naples Italy \\ G. Meccariello \\ Istituto Motori CNR, via Marconi 8 - 80125 Naples Italy
}

\begin{abstract}
In recent years the progress of the automobile industry, in the field of reduction of emissions values, is very remarkable. Nevertheless their evaluation and reduction is a key problem, especially in the cities, that account for more than $50 \%$ of world population. So a correct evaluation of pollutant emissions and fuel consumption by vehicles in real use and precisely geolocated in a road is an important activity and it is still open in the international scientific contexts. A particular attention was given to the slope variability along the streets during each journey performed by the instrumented vehicle. In this paper we deal with the problem of describing a quantitatively approach for the reconstruction of GPS coordinates and altitude, in the context of correlation study between driving cycles / emission / geographical location.
\end{abstract}

Keywords: Air pollution, Driving cycles, GPS signal, Slope variation.

\section{Introduction}

The evaluation of the emission produced by vehicles in correspondence of determined traffic situation in a specific road with specified traffic management rules is generally carried out by multiplying emission factors per vehicle activity, obviously considering different vehicle types.

The problem is thus, in principle, defined when the following information is available:

- Road characteristics (number of lanes, type of pavements, crossing...)

- Traffic management rules (traffic lights, parking, speed limits..)

- Vehicle composition (fleet composition, vehicle age distribution,...) and activity.

- Vehicle flow and density, congestion level of road.

In recent years we are seeing positive results, but on a national scale are still far from achieving this goal. In the case of Italy, factors that are yet contributing negatively to this situation are so different. To obtain the emission factors consolidated methods make reference to vehicle mean velocity, which can be easily obtained by vehicle flow and density in the road. In this framework a new statistical approach has been proposed capable to consider more attributes than the simple speed to characterize driving behavior, not only in the determination of driving cycles but also in the emission modeling. In Naples many research programs have been carried out

Livia Della Ragione, Mathematics, University of Naples "Federico II". Istituto Motori National Research Council, Napoli, 80125 Italy (phone: 39-081-7177175; fax: 39-081-2396097; e-mail: 1.dellaragione@im.cnr.it).

Corresponding author: Giovanni Meccariello, Economics, University of Naples "Federico II". Istituto Motori National Research Council, Napoli, 80125 Italy (phone: 39-081-7177114; fax: 39-081-2396097; e-mail: g.meccariello@im.cnr.it). 
on this subject, whose aim was to determine driving behavior and emission trends. Preliminary results show that if we consider a specific road driving behavior changes and so driving cycles of different characteristics always occur. In this context, it could be interesting to suggest paths based not only on the minimum distance, but on the minimization of fuel consumption as a function of the geomorphological features of the territory. For this purpose, the activities will be aimed at integrating automatically, the commercial digital maps with geomorphological data relating to the real three-dimensional pattern of the road network.

During the development of a research project, an experimental campaign is realized and some results relative to tests performed on road with a Fiat Panda Bipower, (CNG and gasoline powered), and a New Panda Twin Air with auto Start \& Stop system, are compared. Gaseous emissions are measured with Portable Emission Measurement Systems (PEMS) on two different urban routes, in terms of traffic and slope characteristics during in use experiments.

The aim of this paper is to synthesize some considerations about the problem of GPS signal reconstruction, especially for altimetry coordinate, to better analyze and to outline the behavior of low environmental impact vehicles in city traffic situations and in a precise location. Also it could be desirable to perform a quantitative analysis of altimetry to evaluate the slope variability during a path, so this variable can contribute to correlate kinematic behavior with emissions.

\section{Background and Motivation}

In a general approach for the determination of emission factors, developed in our research groups and present in literature and in European research projects, two distinct flows could be considered and evaluated.

One is relative to the development and updating of the emission factors. In this aspect, on road tests have to be performed in different geographical areas to collect experimental data on driving behavior relative to different street networks, traffic conditions and specific features of each geographical areas, with vehicles of different segment and technology. On road data have to be analyzed by statistical methods to determine typical and statistically representative groups of driving cycles and engine (with related accessories) operating conditions. So far, emission modeling is based on measurements performed with vehicles on dynamometer chassis performing driving cycles statically representative of the behavior, considering and simulating engine operating conditions. Applying regression techniques to pollutant data, emission models can be obtained to determine functions relating these data with relevant characteristics of driving cycles, which produce higher and statistically significant effects on environment. In this way, from the experimental work performed on the road it is possible to create a data base of driving cycles and operating conditions. Also from laboratory and on road data acquisition, emission data base is built. Combining these two activities determines the emission factors. Obviously, the experimental work has to be performed continuously to update driving and emission data respect to the upgrading of vehicle technology, considering also other factors affecting emissions as deterioration of used vehicles [1]-[5].

The other research line in this context is relative to the building of relationships between emission, driving behaviour and traffic road network. This link can be created by an effective way by simulation. In fact, flow assignment and micro-simulation models developed recently are capable to determine with good approximation vehicle composition, vehicle flows and vehicle motion in a road network. Micro simulation models are capable to simulate vehicle by vehicle motion considering different driver styles, vehicle types, road characteristics, traffic and parking management rules. 
The link between traffic-road characteristics and driving behavior-emission factors can be built by setting same quantities to cluster driving cycles and to build emission modeling. But to give an added value in terms of the precise geographic position, a problem that cannot be overlooked is the reconstruction of the GPS signal. Today this signal is widely acquired on board during car trip and therefore the correct geolocation of a driving cycle both in terms of latitude and longitude than altimetry is a very interesting issue to be investigated.

Following the general approach, driving cycles have been determined without any conditioning of data respect to road network, but keeping the information on which road the driving data have been detected, when applicable.

The method utilized to determine the driving cycles is based on sequence characterization (a sequence is the part of motion of a car between two successive stops). Variables characterizing each sequence are relative to car speed and acceleration, gear usage and sequence time and length. Clustering of sequences by multivariate statistical analysis give the basic information to cut automatically driving cycles from the real car velocity profile detected on the road. A new cycle starts when a sequence belonging to a different cluster is encountered in the car speed time series.

In the following table variables characterizing sequences are reported. These variables are related to all possible aspects analyzed, both those related to the kinematics of the vehicle and those relating to distributions of acceleration, than those relating to the variability of the gradient of altitude. In terms of kinematic, they refer to speed and acceleration attributes, time duration and length of driving cycle, idling and driving time.

Therefore it could be plausible and it must be investigated that the weight of the variables in creating and grouping driving cycles is different depending on their geographical location, especially when realized paths have frequent slope variations, that is made up by hills and plains.

So in our approach we define and calculate all the variables, and then we use the most appropriate in the statistical analysis also depending on the path achieved in the experimental phase.

Table 1

Variables characterizing kinematic sequence

\begin{tabular}{ll}
\hline Variable & Description \\
\hline $\mathrm{mv}(\mathrm{km} / \mathrm{h})$ & Mean of running speed $(\mathrm{v}>0)]$ \\
$\mathrm{mv} 2\left(\mathrm{~km}^{2} / \mathrm{h}^{2}\right)$ & Mean of square speed $(\mathrm{v}>0)$ \\
$\mathrm{mv} 3\left(\mathrm{~km}^{3} / \mathrm{h}^{3}\right)$ & Mean of cube speed $(\mathrm{v}>0)$ \\
Tral $(\mathrm{s})$ & idling time $\mathrm{v}=0$ in second \\
Trunning $(\mathrm{s})$ & total running time $(\mathrm{v}>0)$ in second \\
Dist $(\mathrm{m})$ & distance covered \\
DS1 $(\%)$ & $\%$ time with delta slope $<-0.70$ meters $(\mathrm{m})$ \\
DS2 $(\%)$ & $\%$ time $-0.70<=$ delta slope $<-0.20$ meters $(\mathrm{m})$ \\
DS3 $(\%)$ & $\%$ time $-0.20<=$ delta slope $<0$ meters $(\mathrm{m})$ \\
DS4 $(\%)$ & $\%$ time $0<=$ delta slope $<0.20$ meters $(\mathrm{m})$ \\
DS5 $(\%)$ & $\%$ time speed $>60$ \\
DS6 $(\%)$ & $\%$ Time with delta slope $>=0.70$ meters $(\mathrm{m})$ \\
Time $(\mathrm{s})$ & Total duration of the sequence $(\mathrm{s})$ \\
$\mathrm{m} \_$vapos $\left(\mathrm{m}^{2} / \mathrm{s}^{3}\right)$ & Mean of instantaneous values of product $(\mathrm{a}(\mathrm{t}) \bullet \mathrm{v}(\mathrm{t}))$ when $\mathrm{v}(\mathrm{t})>0$ and a $(\mathrm{t})>0$ \\
\hline
\end{tabular}


Table 1 continued

\begin{tabular}{ll}
\hline Variable & Description \\
\hline Paccel1 (\%) & \%time with acceleration in range $[-\infty ;-1.4] \mathrm{m} / \mathrm{s}^{2}$ \\
Paccel2 (\%) & \%time with acceleration in range $[-1.4 ;-0.6] \mathrm{m} / \mathrm{s}^{2}$ \\
Paccel3 (\%) & \%time with acceleration in range $[-0.6 ;-0.2] \mathrm{m} / \mathrm{s}^{2}$ \\
Paccel4 (\%) & \%time with acceleration in range $[-0.2 ;+0.2] \mathrm{m} / \mathrm{s}^{2}$ \\
Paccel5 (\%) & \%time with acceleration in range $[+0.2 ;+0.6] \mathrm{m} / \mathrm{s}^{2}$ \\
Paccel6 (\%) & \%time with acceleration in range $[+0.6 ;+1.4] \mathrm{m} / \mathrm{s}^{2}$ \\
Paccel7 (\%) & \%time with acceleration in range $[+1.4 ;+\infty] \mathrm{m} / \mathrm{s}^{2}$ \\
\hline
\end{tabular}

On road tests will be performed in the Naples area were two paths have been selected to sample driving behaviour. Tests will be performed with an instrumented car capable to detect and record, car velocity and position, engine parameters and other parameters. Also pollutant exhaust emissions are sampled by a SEMTECH portable analyzer [6]. They are carbon oxide, nitro oxides, hydrocarbons and carbon dioxide (CO, $\mathrm{NOx}, \mathrm{HC}$ and $\mathrm{CO}_{2}$ respectively). Driving cycles have been obtained by cutting velocity profile recorded on the road by a heuristic rule based on multivariate statistical analysis of kinematic sequences. Driving cycles are clustered into groups to determine typical and statistical car performance in different roads with different experienced traffic conditions. From each group the most representative cycle is determined by discriminant analysis. How to make this selection is a very important matter in the general problem, because on these cycles emission measurements are performed.

\section{Methodology to analyze elevation and gps profiles}

The following diagram illustrates the general layout of the overall approach. The first and third blocks were analyzed in previous work.

The core of the paper concerns the central block, which refers to the reconstruction of the GPS signal with the aim to solve the frequently problems that can arise from the acquisition of this signal in real time [7]-[12].

During the review of the data of the GPS signal, even though the vehicles are equipped with a GPS instrument that returns the values of position and elevation of the path with a $10 \mathrm{~Hz}$ frequency, as a theoretical precision of the instrument; the data for altimetry varies in a range of $+/-10 \mathrm{~ms}$. This change makes it not completely accurate analysis of the slope on the way.

Such errors, for what concerns the coordinates, have occurred in the form of:

- position values sometimes missing;

- misplacement of the vehicle, for example, in areas that are not followed during the journey;

- drift of the position values with the vehicle stationary.

For what concerns instead the elevation errors, they were found:

- changes in short strokes, with slope values are incompatible with reality;

- discrepancies in absolute terms, by comparison with ordinary topographic maps and Google Maps.

So the logical process defined to individuate an algorithm to perform in a mathematical and in an automatic way the signal reconstruction consists in several steps described below. On the first phase, through the Google API (Application Program Interface), it is possible to get the correct map data DTM (Digital Terrain Model) of the theoretical path, on which we performed the real tests. A second phase aims to reconstruct accurate geolocation and slope of the vehicle respect to the GPS signal recorded in real use. To this end, a 
software solution has been implemented in $\mathrm{C \#}$, which should facilitate the correction and completion of the data set of coordinates.

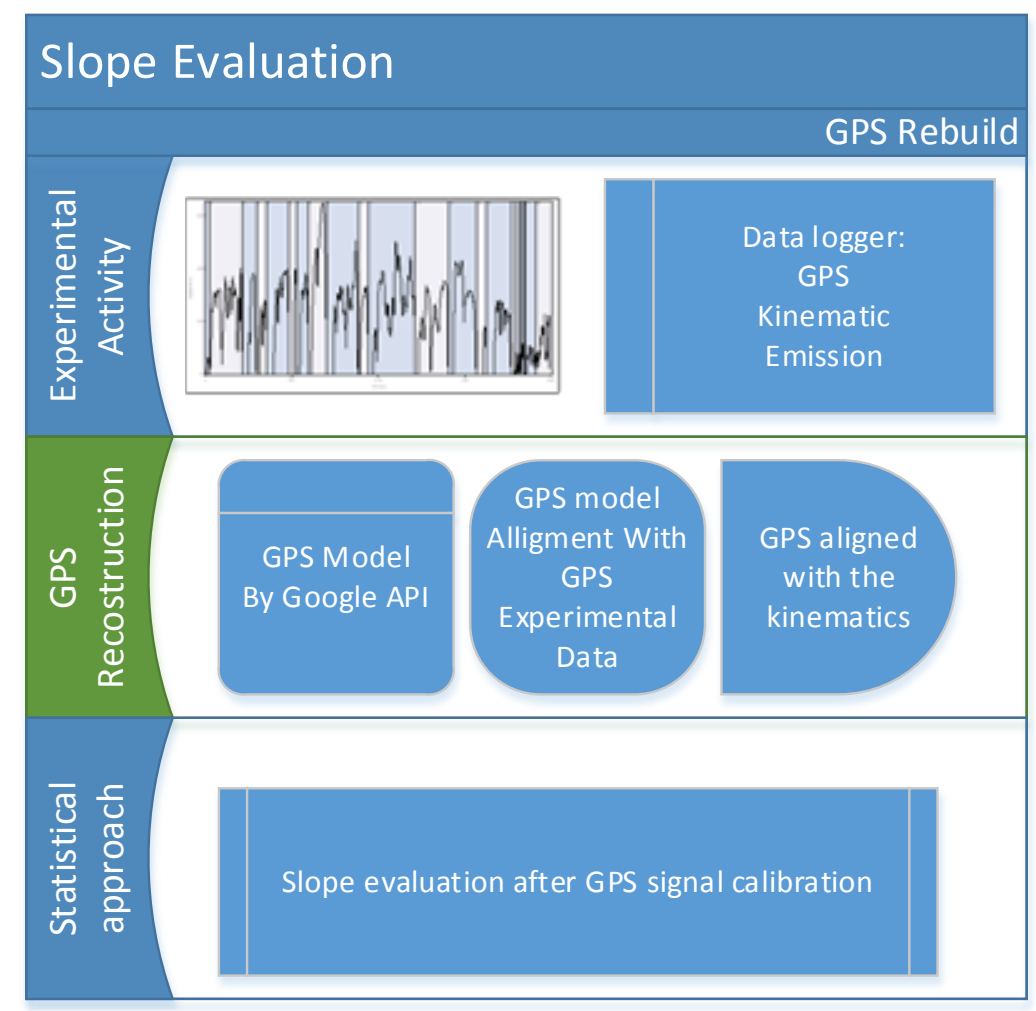

Figure 1. General layout of the approach.

In the reconstruction phases we must consider that the recorded distance is different from the real one, due to the error of the recorded velocity signal especially in the speed range close to 0 .

After data processing which globally consists in steps based on the identification of the beginning and the end of the Experimental Path (EP) and the Model Path (MP), then scale and realign the EP in relation to the MP model, and finally get the correct EP elevation profile. It is aligned with the kinematics and the real distance traveled by the vehicle.

The following sections describe the implementation of the approach for the analysis of the two paths.

\section{Path A}

The path A is located in central Naples predominantly flat of about $22 \mathrm{~km}$, as shown in Fig. 2. In the following analysis only the outward part (about $11 \mathrm{~km}$ ) is taken into account.

The following Figs. 3 and 4 show the graph of Experimental Path A (EP) and the Model Path A (MP) imported through the software. In particular the Fig. 3 shows the EP on a latitude and longitude graph. In the analysis of flat route issues related to the presence of tunnels along the route are highlighted with parts missing in the GPS coordinates. Moreover in the first part of the track there is also a misplacement of the vehicle position.

This has allowed us to understand how the acquired data deviate from the path actually followed, and make the reconstruction of the signals.

Fig. 4 shows the graph of the corresponding Model Path used to match and aligns the GPS data of the EP. 
Fig. 5 shows the elevation profile of the MP (in green) with the linear correction of slope in proximity of the tunnels along the route because the API response gives us the altitude of the hills.

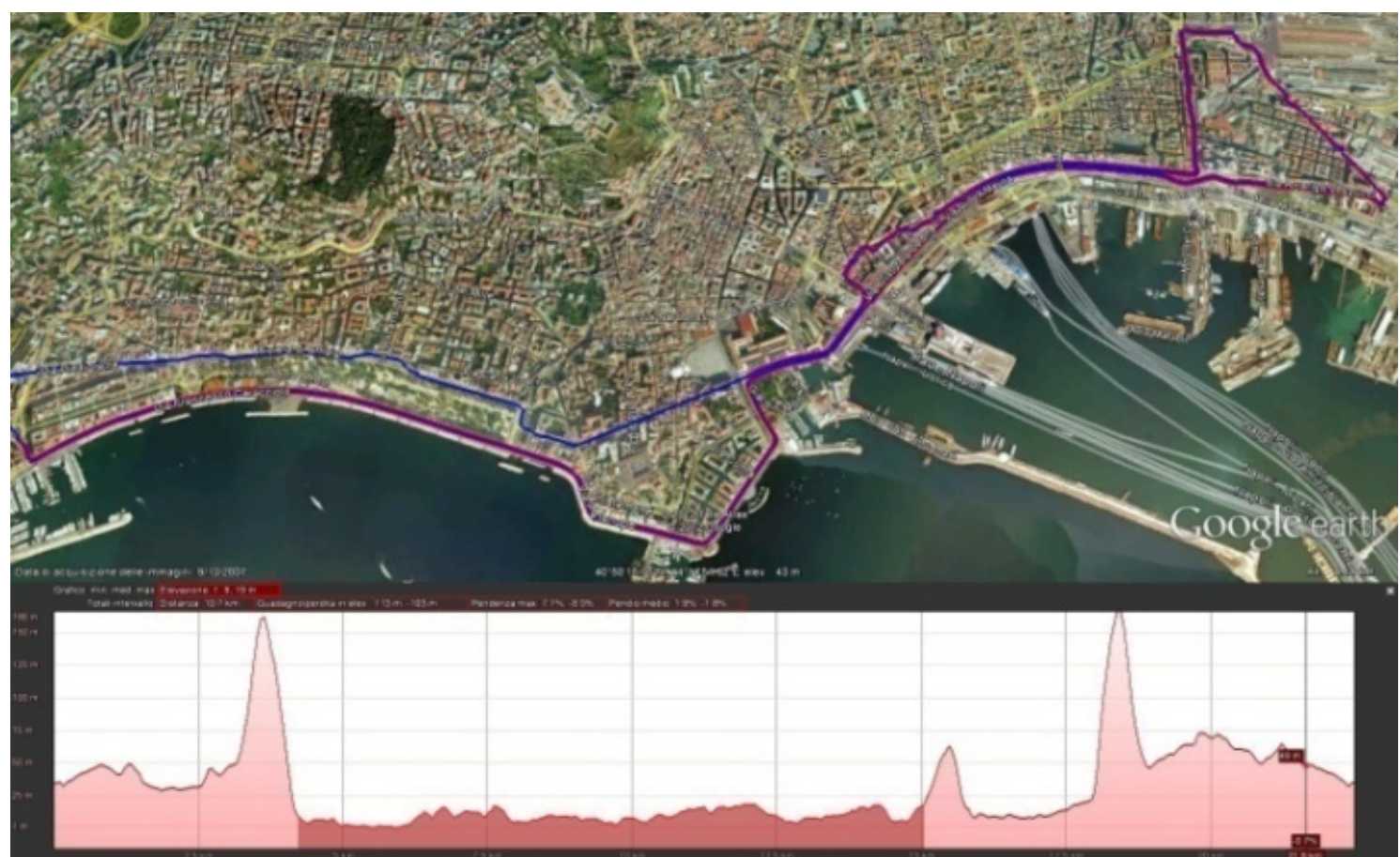

Figure 2. Experimental route: Path A Outward (Istituto Motori- P.za Garibaldi) and return (P.za Garibaldi - Istituto Motori).

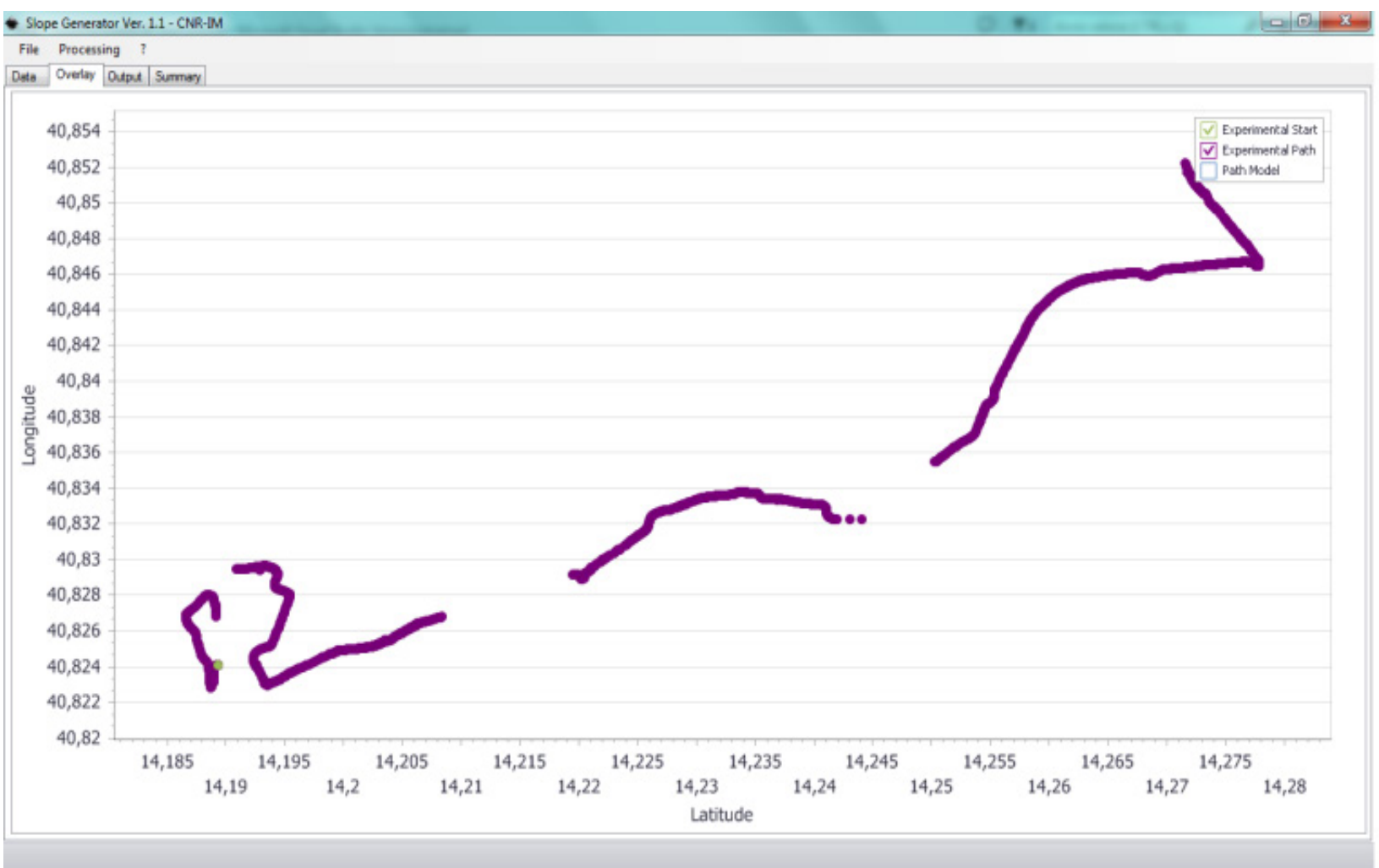

Figure 3. Lat. vs Long. Experimental Path A. 


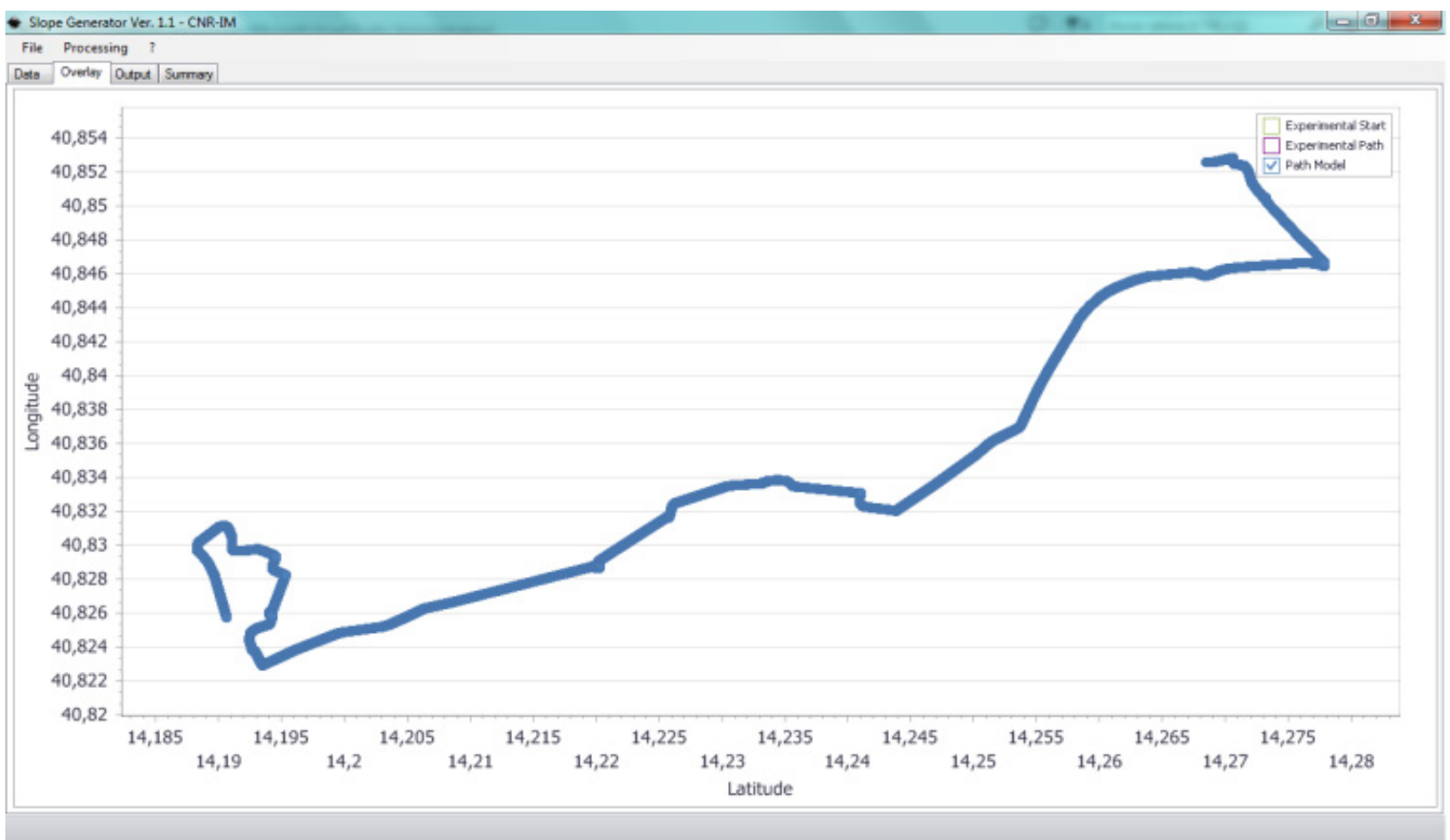

Figure 4. Lat. vs Long. Model Path A.

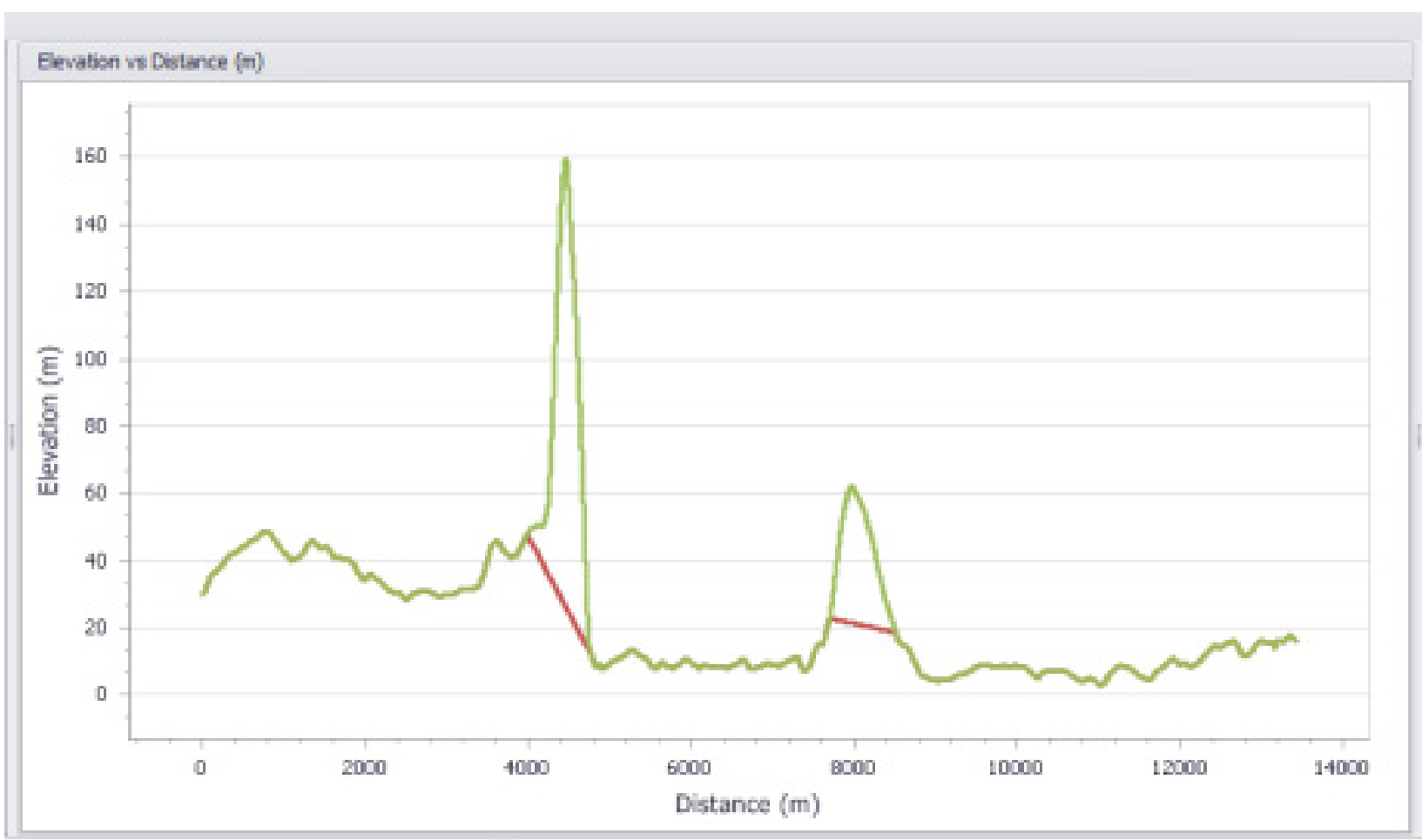

Figure 5. Elevation profile of the Model Path A.

\section{Path B}

The Path B, as shown in Fig. 6, is realized in the hilly area of Naples, with roads with numerous elevation changes. The path length is about $8 \mathrm{~km}$. 


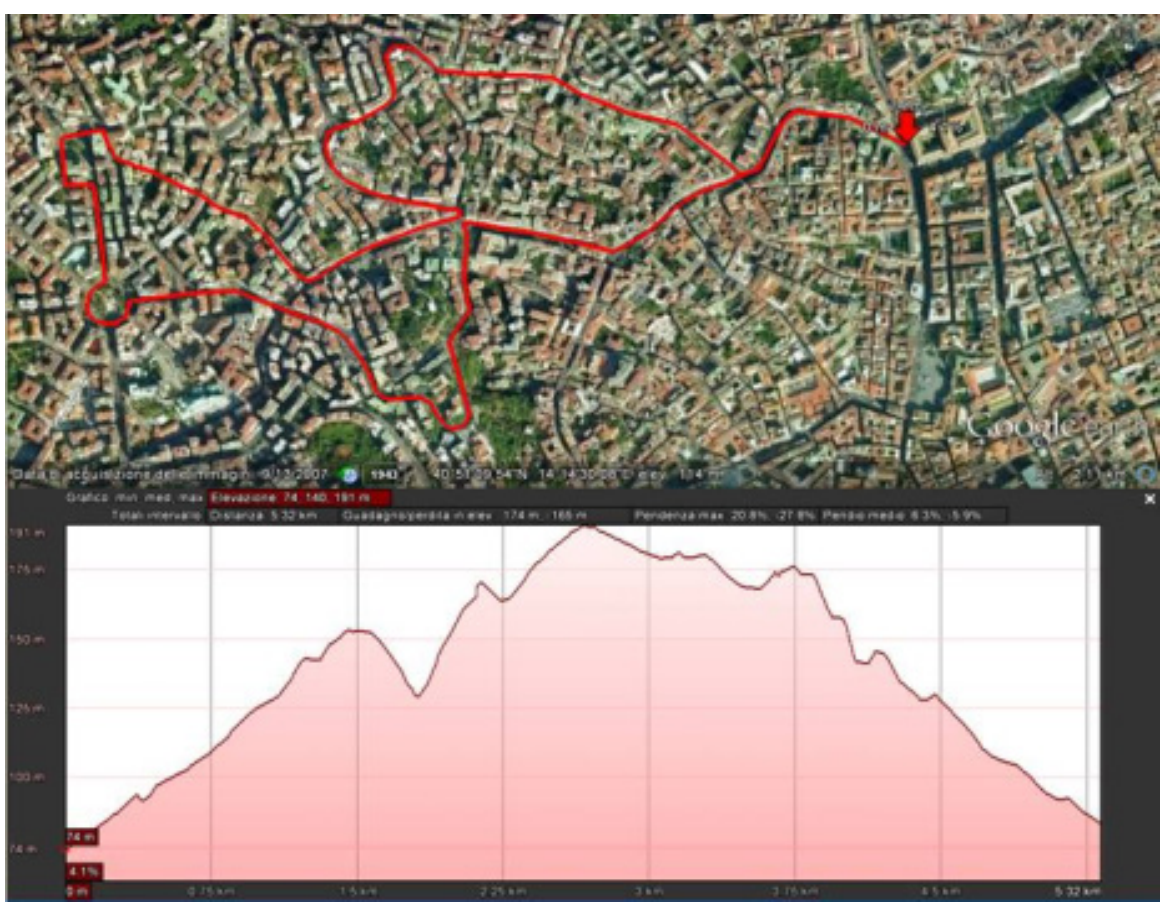

Figure 6. Experimental route: Path B: the hilly area of Naples.

The Figs. 7 and 8 show the graph of Experimental Path B (EP) and the Model Path B (MP) imported through the software. In particular the Fig. 7 shows the EP on a latitude and longitude graph. In the analysis of this path problems can be arise due to continuous changes of elevation, signal drift and the presence of tall buildings leading to the loss of the GPS signal.

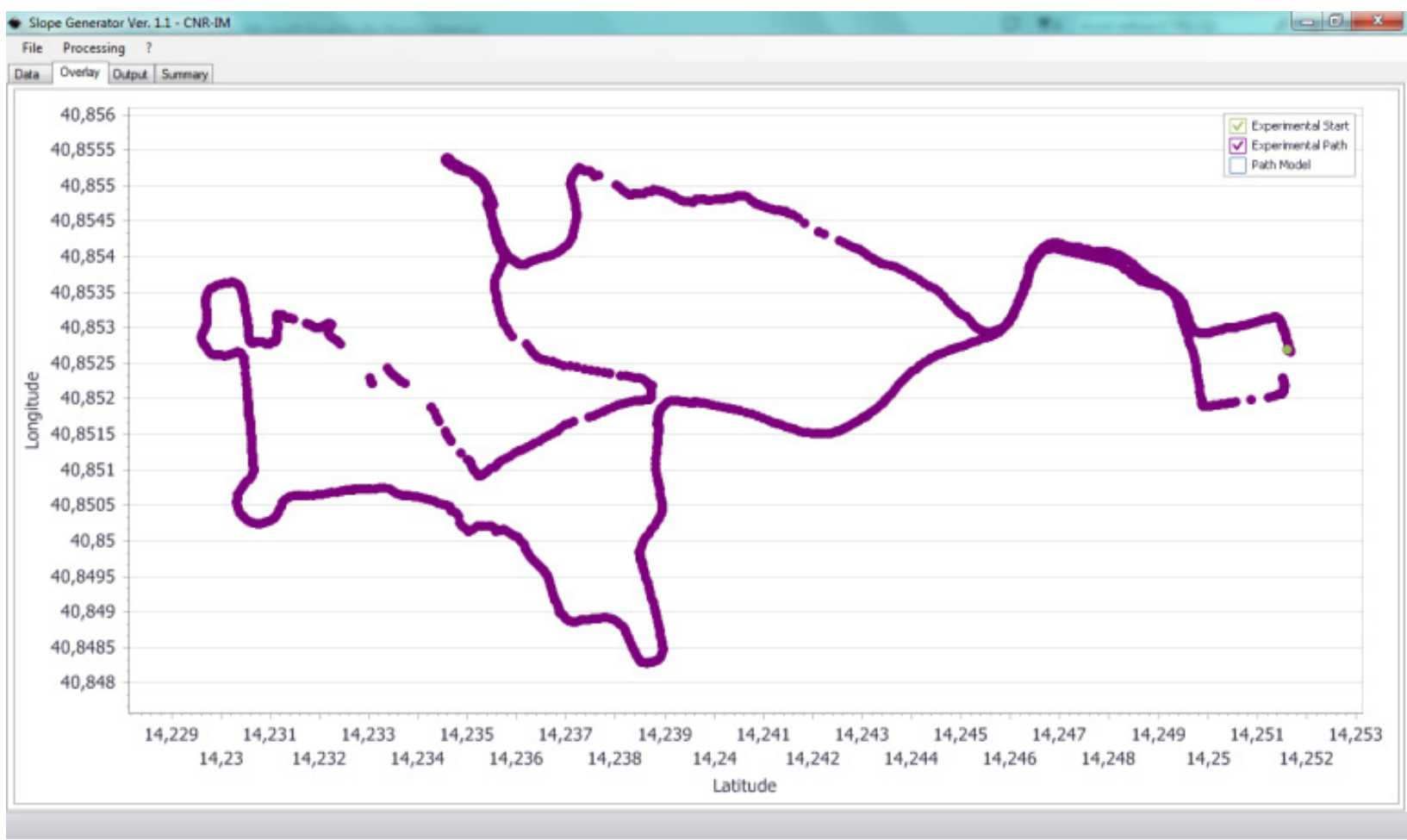

Figure 7. Lat. vs Long. of the Experimental Path B. 
Fig. 8 shows the graph of the corresponding Model Path used to match and aligns the GPS data of the EP.

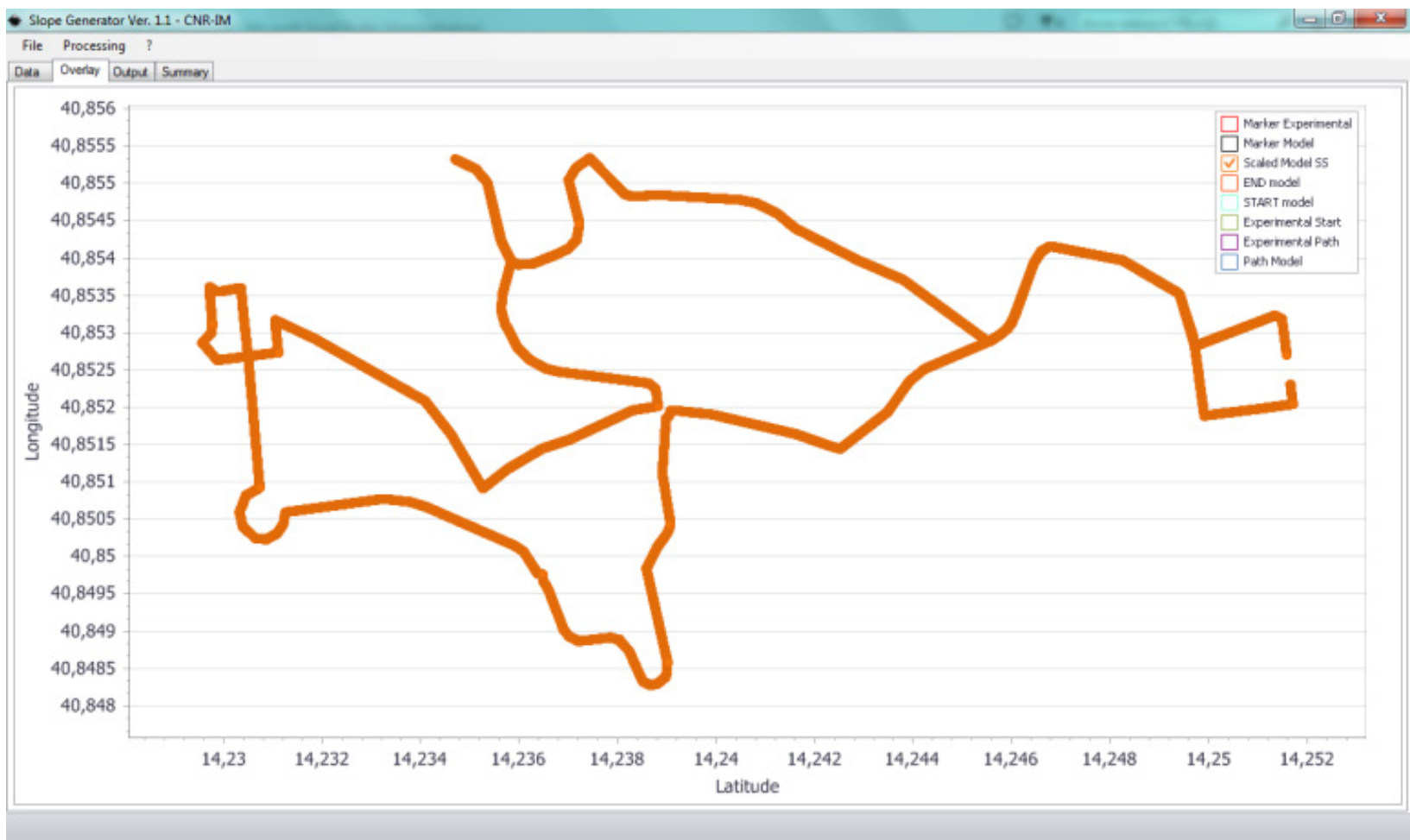

Figure 8. Lat. vs Long. of the Model Path B.

Fig. 9 shows the elevation profile of the MP B obtained through the Google API.

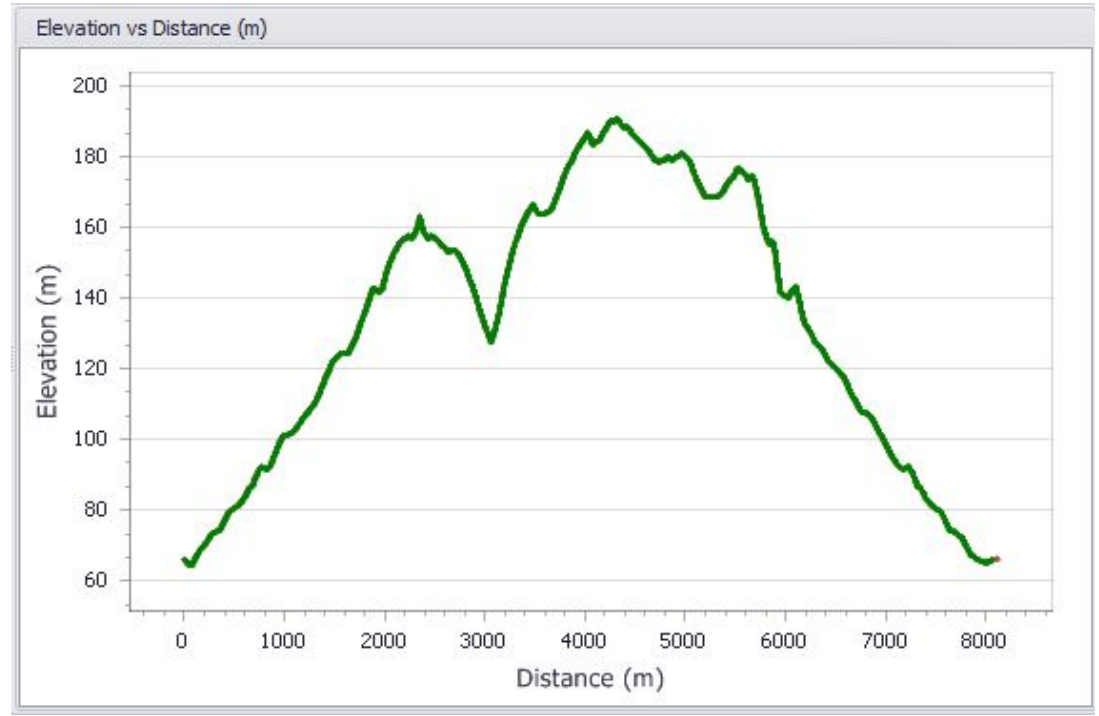

Figure 9. Elevation profile of the Model Path B.

\section{Results}

Applying the algorithm identified, on the experimentation carried out on different days, we can reconstruct automatically, for each trip, all the profiles of the elevations perfectly aligned with the kinematic. 
We have to take in mind that the elevation profile obtained by Google API, shown in Figs. 5 and 9, is speed independent and consequently also the distance calculated. Instead the distance calculated from the real profile is obviously dependent on the speed recorded. In the data processing we must keep in mind that gap. After data processing, which globally consists in several steps based on the identification of the beginning and the end of the EP and MP, scale and realign the EP in relation to the MP model, it is possible to get the correct EP elevation profile (Figs. 10 and 12). It is aligned with the kinematics and the real distance traveled by the vehicle (Figs. 11 and 13).

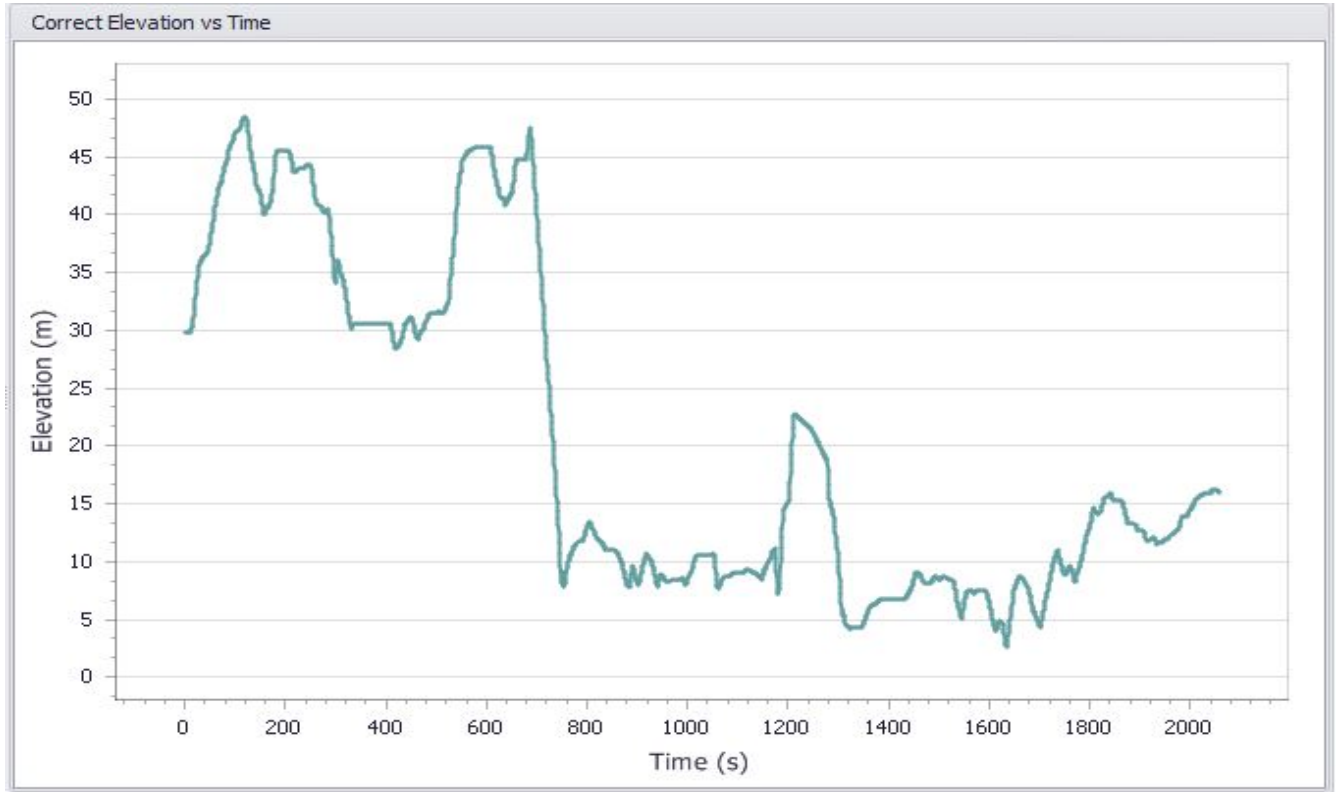

Figure 10. Correct elevation vs time of the Path A.

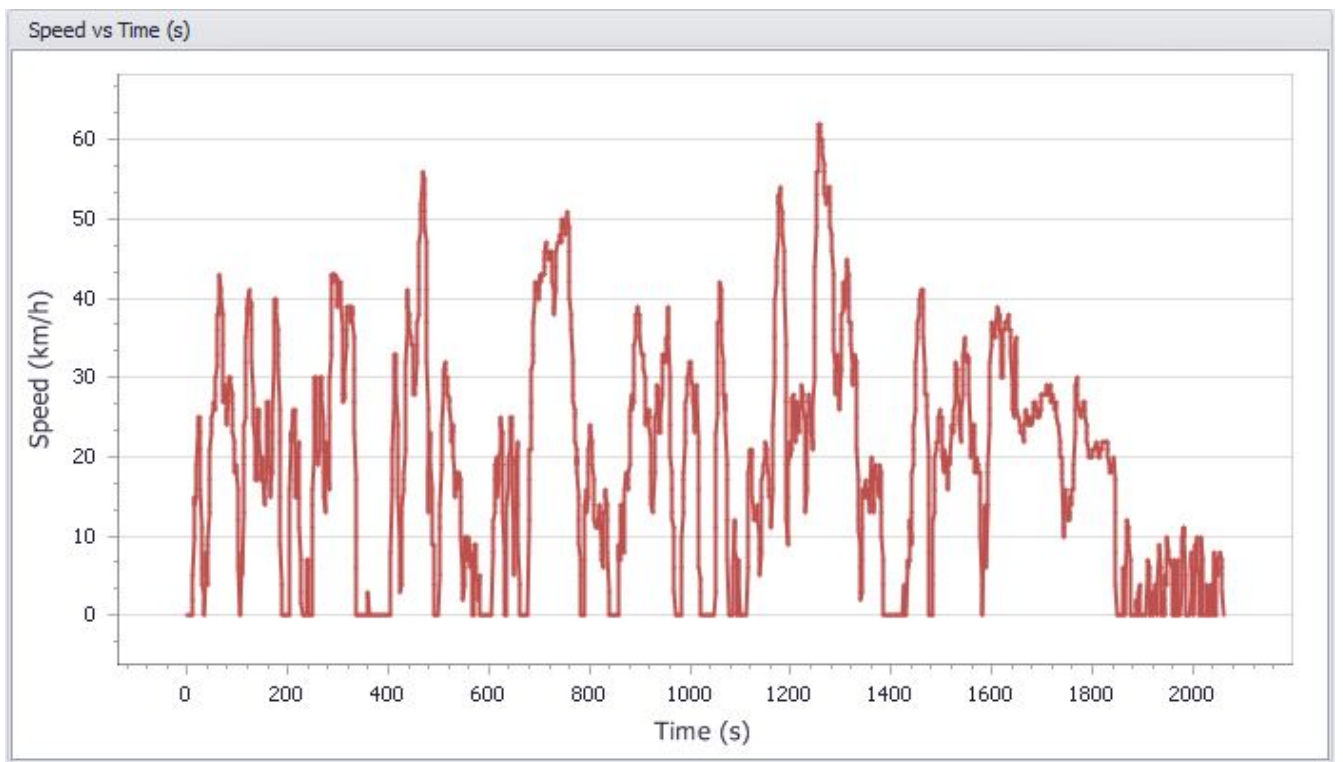

Figure 11. Speed profile of the Path A.

Fig. 10 may be compared to Fig. 5, taking into account that the first one is represented respect to the distance and the other one respect to time. Around the seconds 700 and 1300 where there is the tunnel under the 
hill where the GPS signal was missing or otherwise incorrect, it could be noticed that the altitude is correct.

Fig. 11 shows the corresponding realigned profile of speed versus time.

The proper alignment of the data processing on the Path B is especially noticeable, comparing Figs. 12 and 13 , when the vehicle is stationary around the second 600 and 1200 .

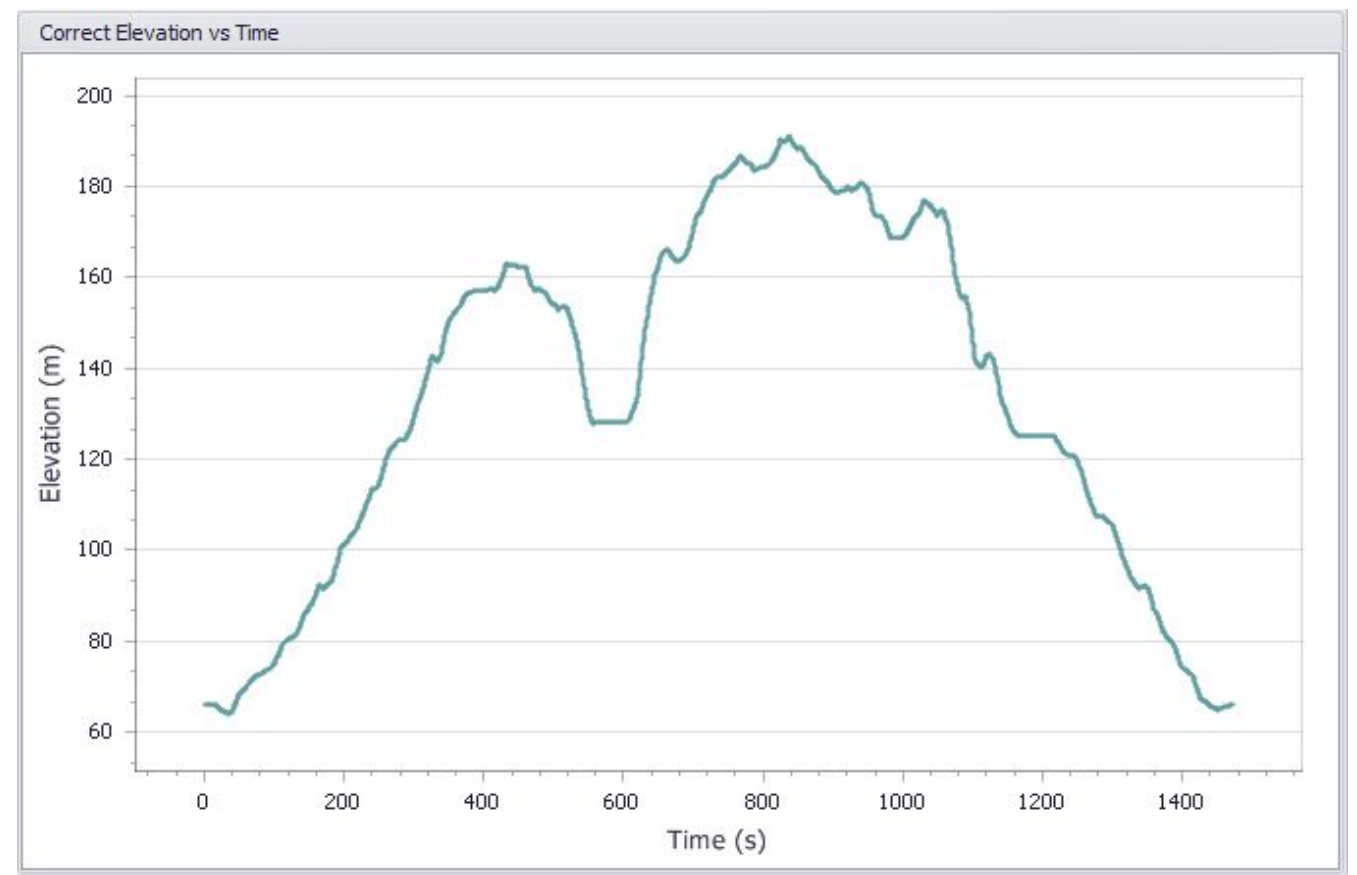

Figure 12. Correct elevation vs time of the Path B.

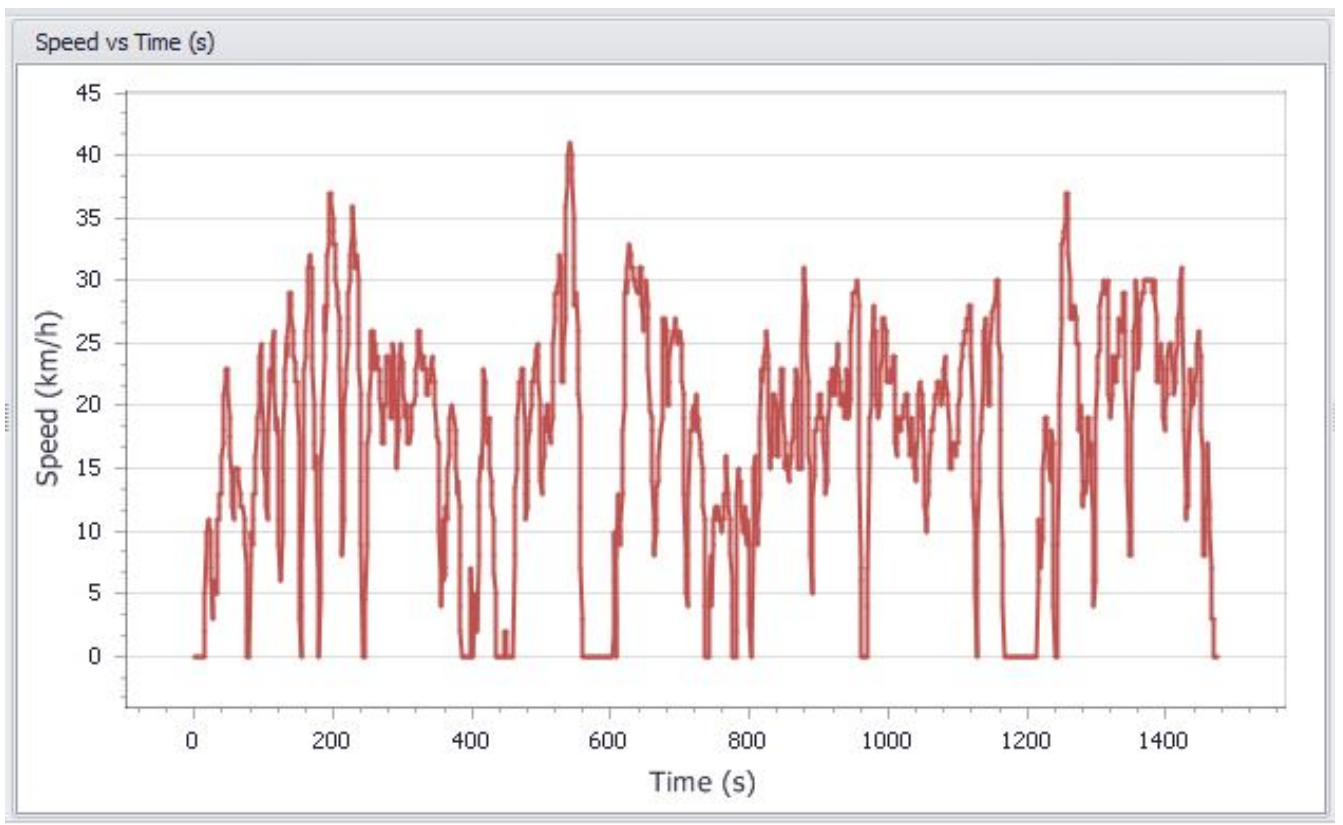

Figure 13. Speed profile of the Path B.

\section{Discussion}

In this paper the authors aim to individuate and to evaluate a procedure to implement the reconstruction of 
the GPS signal during a real experimental campaign. A more precise GPS signal and altimetry value sure help to better perform a quantitative analysis of driving behavior. These signals, in particular altimetry value, may be statistically evaluate to produce variables that describe the variation of the slope and the distance traveled during a trip with greater precision. These variables are among the most significant in kinematic sequences / driving cycles classification above all on a journey in path with many uphill and downhill phases.

Future works attempt to introduce these variables into a new multivariate statistical analysis of driving behavior to evaluate the possibility to statistically analyze the driving behavior to evaluate new clusters of sequences / cycles and to conduct a comparative emissions analysis on sequences with and without these slope variables.

\section{Acknowledgment}

This activity research is realized within a national scientific project PON01_00744 DRIVE IN2. It was carried out by the Istituto Motori of the National Research Council.

\section{References}

Andrè, M. Joumard, R. Vidona, R. Tassela, P. Perret, P., (2006) "Real-world European driving cycles, for measuring pollutant emissions from high- and low-powered cars". Atmospheric Environment, 40 (31), pp. 5944-5953.

Joumard, R. André, J.M. Rapone, M. Zallinger, M. Kljun, N. André, M. Samaras, et al (2007) "Emission factor modelling and database for light vehicle". Artemis deliverable 3. Inrets report, Bron, France, nLTE 0523, p. 237.

Rapone M., Prati M.V., Meccariello G., Della Ragione L., Costagliola M.A., (2005) A Novel Statistical Model For The Evaluation Of Vehicle Emission Factors. Application To A Euro III Gasoline Car Fleet, Proceedings 7th International Conference On Engines For Automobile. Ice2005. Capri, Naples. Sae Paper 2005-24-024.

André M. (2004) "Real-world driving cycles for measuring cars pollutant emissions - Part A : The Artemis European driving cycles". INRETS report, Bron, France, nLTE 0411, 97 p.

Merkisz, J., (2011) "Real Road Tests - Exhaust Emission Results From Passenger Cars", Journal of KONES Powertrain and Transport, Vol. 18, No. 3.

Weiss, M., Bonnel, P., Hummel, R., Urbano, M., Colombo, R., Lanappe, G., Le Lijour, P., Sculati, M.,(2011) "Analyzing on-road emissions of light-duty vehicles with Portable Emission Measurement Systems (PEMS)", JRS scientific and Tecnical Reports, EUR 24697 EN, ISBN 978-92-79-19072-8.

Della Ragione L., Meccariello G., Costagliola M.A., Prati M.V., (2014) Statistical evaluation of slope's effect on real emissions and fuel consumption performed with different cars in Naples urban area, TRA Transport Research Arena 2014 Conference, Paris

Boriboonsomsin K., M. Barth1 (2009). Impacts of Road Grade on Fuel Consumption and Carbon Dioxide Emissions Evidenced by Use of Advanced Navigation Systems. Journal of the Transportation Research Board, Volume 2139 / 2009, Pages 21-30

Frey H.C., K. Zhang, N.M. Rouphail (2008). Fuel Use and Emissions Comparisons for Alternative Routes, Time of Day, Road Grade, and vehicle Based on In-Use Measurements. Environ. Sci. Technol. 2008, 42, 2483-2489

Hong S. Bae1, Jihan Ryu and J. Christian Gerdes (2005). Road Grade and Vehicle Parameter Estimation for Longitudinal Control Using GPS. Proceedings, Annual Meeting of the Air \& Waste Management Association, Minneapolis, MN

Vandana Patel, Pankaj Shukla and Mithilesh Kumar, (2011) "Improved Circular Correlation Methods for Acquisition in Software GPS Receiver", Computer Technology and Application 2 (2011) 389-395.

V. C. Drosos, C. Malesios, (2012), Measuring the Accuracy and Precision of the Garmin GPS Positioning in Forested Areas: A Case Study in Taxiarchis-Vrastama University Forest, Journal of Environmental Science and Engineering B 1 (2012) 566-576, Formerly part of Journal of Environmental Science and Engineering, ISSN 1934-8932. 